\title{
Research on Earthwork Calculation Based on TIN Model
}

\author{
Wen Yongxiao ${ }^{1}$, Yuan Xitun ${ }^{1}$, Yang Xiongfei ${ }^{1}$, Zhang Hang, ${ }^{1} \mathrm{Han}_{\mathrm{Qi}}{ }^{1}$ \\ ${ }^{1}$ Xi'an University of Science and Technology,710000, Xi'an City
}

\begin{abstract}
In actual engineering construction, the calculation of earthwork directly affects the cost budget of the project and the selection of the optimal plan. Therefore, the calculation accuracy and efficiency of the earthwork are very important. This article introduces the construction principle of the TIN model. Based on the TIN model, the earthwork calculation is carried out by the triangular prism method. The data of a fill-excavation balance project in a rugged mountainous area is selected, and under different conditions, the TIN network method and the square grid method are used to calculate the earthwork, and the results are compared and analyzed with accuracy. Estimate. After comparative analysis, it is found that the earthwork calculation using the TIN network method is simple and cheap, and the calculation result is more accurate. It is better than the traditional square grid method as a whole, and can be used in the actual engineering earthwork calculation.
\end{abstract}

\section{Introduction}

With the rapid economic development, nowadays, there are more and more engineering construction projects at home and abroad, and there are many issues that need to be considered in each engineering construction project. Among them, earthwork calculation is an unavoidable and vital part. Traditional earthwork calculation methods mainly include section method, square grid method. Nowadays, the rapid development of computer technology has also promoted the development of surveying and mapping. Computers have brought us more means of data collection, extraction and processing. At the same time, they have also brought us more considerations about the calculation method of earthwork. We can combine traditional earthwork calculation methods with Digital Elevation Model (DEM) to achieve DEM-based engineering earthwork calculations. Among them, the TIN network method can be generally applied to flat or rugged engineering areas. Therefore, the TIN network method has become a better choice for the calculation method of engineering earthwork.

In terms of research status, Deng Xiaodong ${ }^{[1]}$ used two different cases to compare the calculation results, compared the accuracy of the calculation results of several methods, and discussed their applicable terrain conditions and scope of application. Yuan Jing ${ }^{[2]}$ combined with MATLAB and AuToCAD2004 software, uses the three-dimensional coordinate data of the sampling points to generate the triangle vertex index file in the TIN network in MATLAB, and then uses the three-dimensional graphics drawing function of AuToCAD to establish the terrain entity model, and by calculating the volume of the model, the amount of earthwork under the terrain can be obtained.

\section{TIN network method earthwork calculation principle}

\subsection{TIN network model}

The Triangulated Irregular Network (TIN) model uses a series of connected triangles to fit the ground or other irregular surfaces. This irregular surface is composed of a series of triangles, which are continuous, and each triangle is generated by a series of irregular data points. In the process of constructing a TIN network, nodes, edges and faces are usually used as the basic units to build a TIN network.

When building a TIN network, three principles must be followed for the establishment of each triangle: each triangle is as close as possible to an equilateral triangle, the three vertices forming the triangle must be the closest, and the triangle network must be unique. Only in this way can the TIN network be as close as possible to the actual terrain surface. At the same time, the triangulation criterion of TIN is also needed, that is, the conditions that must be used for triangle generation in TIN, mainly include: empty circumscribed circle criterion, maximum minimum angle criterion, shortest distance and criterion, maximum opening angle criterion, area ratio Guidelines, diagonal guidelines. At present, the most widely used subdivision method is Delaunay triangulation ${ }^{[3]}$, which must meet the following two important criteria:

(1) Hollow circle characteristics. The Delaunay triangle network is unique (any four points cannot be a circle), and there will be no other points within the circumcircle of any triangle in the Delaunay triangle network. As shown below: 


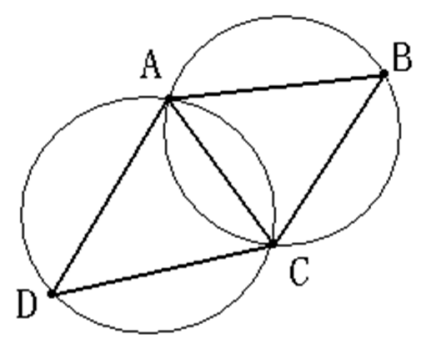

Figure 1 Schematic diagram of empty circle characteristics

(2) Maximize the minimum angle characteristic. Among the possible triangulations formed by the scattered point set, the smallest angle of the triangle formed by the Delaunay triangulation is the largest. In this sense, the Delaunay triangulation is the "closest to regularization" triangulation. Specifically, it means that the diagonals of a convex quadrilateral formed by two adjacent triangles, after mutual exchange, the smallest angle of the six internal angles no longer increases. As shown below:

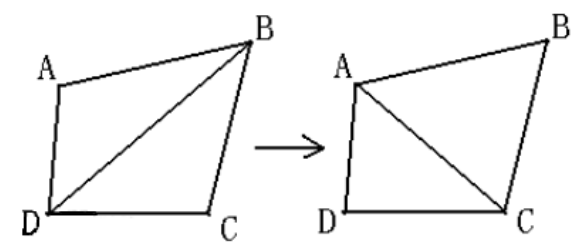

Figure 2 Schematic diagram of maximized minimum angle characteristics

\subsection{TIN network model construction}

The establishment of TIN network should consider the choice of triangulation network. Among all possible triangulations, the Delaunay triangulation is more commonly used in terrain fitting. The Delaunay triangulation can avoid the generation of triangles with too small acute angles when the discrete points are evenly distributed. It also performs best in terms of integration, so people generally build TIN as a Delaunay triangulation. The methods of establishing Delaunay triangulation mainly include segmentation and merging algorithm, triangulation growth algorithm and point-by-point insertion algorithm. This paper uses triangulation growth algorithm to generate Delaunay triangulation $^{[4]}$.

The triangulation growth algorithm was proposed by Brassel and Reif in 1979. This algorithm refers to forming a triangle from a triangular point roughly in the center of the area, and then using this triangle as the basis to continuously expand outwards until it forms a The overall triangulation that contains all the original data points. The specific steps are as follows ${ }^{[5]}$ :

(1) Select a data point A near the geometric center point of the area containing all the original data points, and then find the closest data point $\mathrm{B}$ to the point $\mathrm{A}$, and connect $\mathrm{A}$ and $\mathrm{B}$ to form the initial baseline. Then use the empty circumscribed circle criterion to find the data point $\mathrm{C}$ that meets the conditions as the third point. The three points $\mathrm{A}, \mathrm{B}$, and $\mathrm{C}$ form the initial triangle.
(2) Taking the three sides of the initial triangle as the starting sides, again using the empty external criterion to continue to find the next data point that meets the requirements. In this way, we have found three other data points that meet the requirements. In this process, it should be noted that any same side can be used twice at most, otherwise the new triangle generated will be invalid.

(3) Repeat the steps of the second step until all the baselines are processed and a complete triangular network is formed.

At this point, the establishment of the TIN network has been completed.

\subsection{Earthwork calculation of TIN network}

The basic steps of the earthwork calculation based on the TIN network are: first calculate the earthwork of each irregular triangle constituted by the column, and then add the earthwork under all the triangular columns in the area to obtain the area The total earthwork volume based on the TIN net.

For the calculation of the earthwork volume of the column body formed by each triangle, considering the actual situation ${ }^{[6]}$, we can divide it into the following three situations according to the relationship between the triangle surface and the design elevation plane ${ }^{[7]}$ :

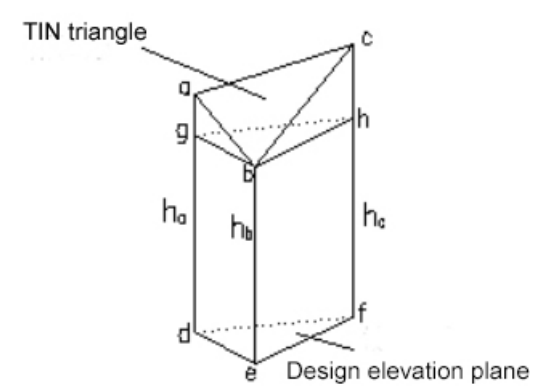

Figure 3 Schematic diagram of excavation

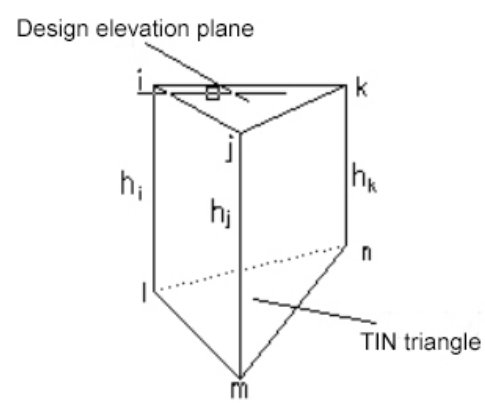

Figure 4 Sketch map of filling 


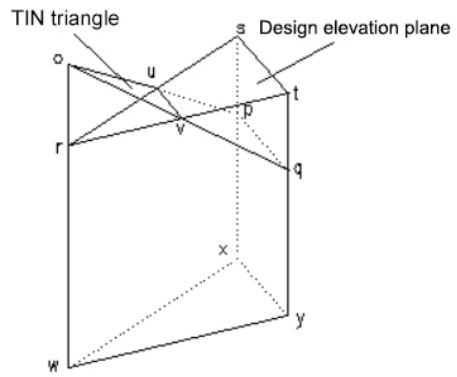

Figure 5 Schematic diagram of filling and excavation

The first type: as shown in Figure 3, the triangle abc is a TIN triangle, the triangle def is the design elevation plane, and ha, hb, and hc are the absolute values of the difference between the elevation of the three points $a, b$, and $\mathrm{c}$ and the design elevation. At this time, the three vertices of the TIN triangle are all higher than the design elevation value, and the earthwork calculated at this time is the excavation. The amount of excavation is the volume of the triangular prism, and the calculation formula is:

$$
V_{d i g}=\frac{\left(h_{a}+h_{b}+h_{c}\right)}{3} S_{d e f}
$$

The second type: As shown in Figure 4, the triangle ijk is the design elevation plane, the triangle $1 \mathrm{mn}$ is the TIN triangle, and hi, hj, and hk are the absolute values of the difference between the elevation of the three points 1 , $\mathrm{m}$, and $\mathrm{n}$ and the design elevation. At this time, the three vertices of the TIN triangle are all lower than the design elevation value, and the earthwork calculated at this time is the fill. The filling amount is the volume of the triangular prism, and the calculation formula is:

$$
V_{f i l l}=\frac{\left(h_{i}+h_{j}+h_{k}\right)}{3} S_{i j k}
$$

The third type: as shown in Figure 5, the triangle opq is a TIN triangle, the triangle rst is the design elevation plane, the line uv is the intersection of these two planes, and the side st is parallel to the side pq, and the side uv is parallel to the surface spqt. In this case, one vertex of the TIN triangle is higher than the design elevation value, and two vertices are lower than the design elevation value. At this time, the amount of earthwork needs to be calculated by the excavation part and the filling part.

The calculation of the excavation part, that is, the volume of the triangular pyramid o-ruv, the calculation formula is:

$$
V_{\text {dig }} \equiv \frac{1}{3} S_{\text {ruv }} h_{1}
$$

In the above formula, $\mathrm{h} 1$ is the distance from o to the ruv surface.

The filling part is calculated, that is, the volume of the wedge-shaped body usp-vtq, the calculation formula is:

$$
V_{\text {fill }}=\frac{D_{t q} h_{2}}{6}\left(2 D_{s t}+D_{u v}\right)
$$

Among them, $\mathrm{h} 2$ is the distance from the side uv to its parallel plane svqt, and Dtq, Dst, and Duv are the distances from point $t$ to point $q$, point $s$ to point $t$, and point $\mathrm{u}$ to point $\mathrm{v}$, respectively.

The sum of the above two parts of earthwork is the earthwork calculated in this case. In actual engineering, it is also possible that one vertex of the TIN triangle is lower than the design elevation value, and two vertices are higher than the design elevation value. The amount of filling at this time should be the volume of a triangular pyramid, and the amount of excavation is the volume of the wedge-shaped body. The calculation steps are similar to the above method, and will not be repeated here.

At this point, the earthwork calculation under a TIN triangulation is completed, and the earthwork of all the prisms formed by irregular triangles is calculated by adding up, and the sum is the total earthwork in the area.

\section{Test}

Using the data of a fill-excavation balance project in a rugged mountainous area, the earthwork calculation was carried out with the TIN net method and the square grid method respectively. The specific results are compared as shown in Table 1:

Table 1 Under uneven terrain conditions, TIN and square gridsEarthwork calculation results comparison

\begin{tabular}{lllll}
\hline $\begin{array}{c}\text { Calculation } \\
\text { method }\end{array}$ & $\begin{array}{l}\text { Design } \\
\text { elevation }\end{array}$ & $\begin{array}{l}\text { Number of } \\
\text { excavations }\end{array}$ & $\begin{array}{c}\text { Number of } \\
\text { fills }\end{array}$ & $\begin{array}{l}\text { Poor fill } \\
\text { and } \\
\text { excavation }\end{array}$ \\
\hline TIN net & 40.456 & 363617 & 366535 & 2918 \\
$\begin{array}{c}\text { Square } \\
\text { grid }\end{array}$ & 40.717 & 362504 & 374139 & 11635 \\
\hline
\end{tabular}

Under the principle of the balance between fill and excavation, if the average of the number of fill and excavation is the most or value, the relative accuracy of the two methods can be calculated ${ }^{[8]}$. The relative accuracy under the TIN method is $0.8 \%$, while the relative accuracy under the square grid is $3.16 \%$. After comparison, it can be found that the TIN mesh method can determine the design elevation and earthwork more accurately than the traditional square mesh method, and the final calculation result will be more accurate.

In addition, a certain abandoned vegetable field is used for calculation, and the field in this area is relatively flat. TIN net method and square grid method are used to calculate earthwork, and the results are shown in Table 2: 
Table 2 Comparison of earthwork calculation results of TIN net and square grid under the condition of flat terrain

\begin{tabular}{lllll}
\hline $\begin{array}{l}\text { Calculation } \\
\text { method }\end{array}$ & $\begin{array}{l}\text { Design } \\
\text { elevation }\end{array}$ & $\begin{array}{l}\text { Number of } \\
\text { excavations }\end{array}$ & $\begin{array}{l}\text { Number of } \\
\text { fills }\end{array}$ & $\begin{array}{l}\text { Total number } \\
\text { of fills and } \\
\text { excavations }\end{array}$ \\
\hline TIN net & 23.62 & 23199.3 & 1028.6 & 24227.9 \\
Square grid & 23364.6 & 1089.8 & 24454.4 \\
\hline
\end{tabular}

After comparison, when the terrain is flat, there is no big difference in the total amount of earthwork calculated under the two methods, but the calculation of the amount of earthwork using the TIN network method is simple and cheap, and the calculation result is more accurate, which can save time, cost and Calculating the cost helps to advance the progress of the project.

\section{Results \& Discussion}

Through the step-by-step analysis of the construction process of the earthwork calculation model based on the TIN network (triangular irregular network) and the subsequent earthwork calculation process, the final calculation accuracy can be analyzed [] as follows:

(1) Theoretically, the earthwork calculation method based on the TIN network is suitable for any situation in actual engineering. When the terrain is relatively flat, this method is similar to the traditional square grid method to calculate the earthwork and is closer to equilibrium. value.

(2) The calculation accuracy of the earthwork calculated by this method mainly depends on the density and accuracy of the original data points. Both the design elevation and the accuracy of the original data point coordinates affect the accuracy of the final calculation result. However, the influence of elevation accuracy on the calculation result is greater than the influence of coordinate accuracy on the calculation result.

(3) In summary, in order to improve the accuracy of earthwork calculation under this method, the main measures that can be taken are: when constructing the triangulation network, determine the correct triangle connection; appropriately increase the density of elevation points; Improve the accuracy of sampling points.

\section{Conclusions}

In the earthwork calculation of the TIN model, the factors that affect the accuracy are mainly in the two parts of model construction and subsequent calculations. When constructing the DEM model, when it comes to interpolation, you should consider the choice of interpolation method, the search of reference points, etc. Doing these steps can ensure the accuracy of the final calculation result to a certain extent. In the calculation process after modeling, you should pay attention to factors such as sampling density, elevation value and location of each point, and these factors will also affect the accuracy of the final result. Using the TIN network method for earthwork calculation, a technology can not only speed up the progress of the project, but also help to improve the accuracy of statistical calculations.

\section{References}

1. Deng Xiaodong, Zhang Chunkang, Cao Tingting, Liu Zhiyong. Several methods of earthwork calculation and accuracy analysis [J]. Mine Surveying, 2019, 47(06): 67-70.

2. Yuan Jing. Earthwork calculation method based on irregular triangulation network $[\mathrm{J}]$. Technical Equipment of Surveying and Mapping, 2008(04): 25-28.

3. Yu Jie, Lu Pin, Zheng Changwen. Comparative study on Delaunay triangulation method construction[J]. Journal of Image and Graphics, 2010, 15(08): 1158-1167.

4. Xi Jingzhi. Optimization analysis and application research of engineering earthwork calculation method based on DEM [D]. Chongqing Jiaotong University, 2013.

5. Chen Su. Comparison of several algorithms for building TIN [J]. Fujian Architecture, 2011(12): 102-104.

6. Liu Guohua, Zhang Yunwen, Zhou Xinshui. Research on Earthwork Calculation Algorithm Based on TIN $[\mathrm{J}]$. Jiangxi Surveying and Mapping, 2006(02): 39-40.

7. Chen Nanxiang, Li Dandan, Li Yaohui, et al. Research and application of earthwork calculation method based on TIN model[J]. Anhui Agricultural Sciences, 2013, (26): 10870-10873. DOI:10.3969/j.issn.0517- 6611.2013.26.112.

8. Surveying Adjustment Subject Group, School of Surveying and Mapping, Wuhan University, Error Theory and Surveying Adjustment Foundation. Second Edition [M], Wuhan University Press, 2009. 